\title{
The Effect of FDG-PET/CT on Clinical Prognostic Staging of Breast Cancer Patients being Planned Neoadjuvant Treatment
}

\author{
Eda Tanrikulu Simsek ${ }^{1}$, Ezgi Coban ${ }^{1}$, Elif Atag ${ }^{1}$, Serkan Gungor ${ }^{2}$, Fugen Aker ${ }^{3}$ and Murat Sari ${ }^{1}$ \\ ${ }^{1}$ Department of Medical Oncology, Haydarpasa Numune Training and Research Hospital, Istanbul, Turkey \\ ${ }^{2}$ Department of Nuclear Medicine, Medeniyet University Hospital, Istanbul, Turkey \\ ${ }^{3}$ Department of Pathology, Haydarpasa Numune Training, and Research Hospital, Istanbul, Turkey
}

\begin{abstract}
Objective: To evaluate the effect of FDG-PET/CT in the radiological imaging of breast cancer (BC) patients planned for neoadjuvant treatment (NAT), on the clinical prognostic stage (CPS).

Study Design: A descriptive study.

Place and Duration of Study: Haydarpasa Numune Training and Research Hospital, Istanbul, Turkey, between June 2014 and September 2020.

Methodology: Consecutive patients with stage I-III breast cancer (BC) who were planned for neoadjuvant treatment (NAT). The distribution of CPS detected by both conventional radiological methods (c-CPS) and FDG-PET/CT (PET-CPS) were compared.

Results: Significant upstaging on CPS was detected with the addition of FDG-PET/CT to conventional imaging methods in $25 / 121(20.7 \%)$ patients $(p<0.001)$. In the c-CPS stage, IB, IIA, IIB, IIIA, IIIB patients, the stage change rate was $22.7 \%, 28.6 \%$, $37.5 \%, 50 \%$, and $9.1 \%$, respectively. There was no change in patients with C-CPS stage IA and IIIC. There was a significant change in the $c N$ stages $(p<0.001)$, while no significant change was detected in the cT stages of the patients $(p=0.180)$. Upstaging was detected in $5 / 16(6.3 \%, p=0.034), 14 / 71(19.7 \%, p<0.01), 15 / 30(50 \%, p<0.01)$ of initially cN $0,1,2$ patients, respectively $(p<0.001)$.

Conclusion: The change in CPS was due to nodal upstaging. The effectiveness of including FDG-PET/CT in the initial radiological imaging in patients planned for NAT should be evaluated with prospective studies evaluating treatment choices to be used in NAT.
\end{abstract}

Key Words: PET scan, Breast cancer, Positron emission tomography, Neoadjuvant treatment, Cancer staging, Staging system, TNM.

How to cite this article: Simsek ET, Coban E, Atag E, Gungor S, Aker F, Sari M. The Effect of FDG-PET/CT on Clinical Prognostic Staging of Breast Cancer Patients being Planned Neoadjuvant Treatment. J Coll Physicians Surg Pak 2022; 32(02):181-185.

\section{INTRODUCTION}

According to 2020 data, breast cancer (BC) has surpassed lung cancer with 2.3 million new cases $(11.7 \%$ of all newly diagnosed cancer cases) and has become the most common cancer type worldwide. ${ }^{1}$ It ranks $5^{\text {th }}$ among cancer-related causes of death worldwide. ${ }^{1}$ In the management of $B C$, which is a global health problem, the primary goal is to prolong survival with effective treatment while reducing treatment toxicities. In this context, determining the most appropriate treatment for each patient can sometimes be difficult for professionals dealing with $\mathrm{BC}$ treatment.

Correspondence to: Dr. Eda Tanrikulu Simsek, Department of Medical Oncology, Haydarpasa Numune Training and Research Hospital, Istanbul, Turkey

E-mail: edatanrikulu@gmail.com

Received: July 07, 2021; Revised: November 21, 2021;

Accepted: December 10, 2021

DOI: https://doi.org/10.29271/jcpsp.2022.02.181
To optimise treatments, it is thought that new strategies are needed in $\mathrm{BC}$ as in other cancers in determining individualised methods. $^{2}$

Accurate staging is one of the most significant factors that determine the treatment plan in newly diagnosed breast cancer (BC). The staging system we previously used for $\mathrm{BC}$ staging was based on the anatomical features determined by primary tumour size ( $\mathrm{T})$, regional lymph node involvement ( $\mathrm{N}$ ), and the presence of distant metastases. ${ }^{3}$ This staging system did not include the biological characteristics of the tumour that affect $\mathrm{BC}$ prognosis.

The clinical prognostic stage (CPS) was upgraded by the 8th edition of the American Joint Committee on Cancer (AJCC) staging system that included oestrogen receptor (ER), progesterone receptor (PR), grade, and human epidermal growth factor receptor 2 (Her2) status, which are the most important biological features known to affect BC prognosis. ${ }^{4}$ Thus, it has become possible to make more accurate prognostic predictions for patients. $^{5}$ 
Neoadjuvant treatment (NAT) before definitive surgery is a frequently used modality in stage I-III BC. The stage is one of the main determining factors besides the biological characteristics of the tumour, while deciding on the NAT to be applied. Recently, the evaluation of escalation/de-escalation strategies to increase NAT efficiency and/or reduce toxicity is an important research area. ${ }^{6}$ The correct regional staging is critical when deciding on the systemic treatment.

The National Comprehensive Cancer Network (NCCN) recommends 18-fluorodeoxyglucose positron emission tomography/computerised tomography (FDG-PET/CT) as optional at the level of category 2B in the initial workup of patients planned for NAT. ${ }^{7}$ The ESMO similarly suggests the use of FDG-PET/CT in cases, where conventional imaging methods are inconclusive or for staging in high-risk patients. ${ }^{8}$ However, there are data in the literature indicating that FDG-PET/CT can change the anatomical stage determined by conventional methods in early and locally advanced stage BC, especially as it more accurately shows regional nodal spread. ${ }^{9-11}$

Today, the use of CPS is recommended for the staging of all BC patients when ER, PR, Her2, and grade information are available. ${ }^{7}$ To date, studies using NAT in BC have been based on anatomical stages. There was no study in the literature related to NAT planning, depending on the CPS to the best of authors' knowledge. Therefore, this study is aimed to evaluate whether FDG-PET /CT performed in addition to conventional methods alters the stage of locoregional CPS in patients who were scheduled NAT.

\section{METHODOLOGY}

All consecutive patients scheduled NAT for stage I- III BC were examined at Hospital between June 2014 and September 2020. The data of 138 patients with $\mathrm{BC}$ was reviewed retrospectively. The 8th edition of the American Joint Committee on Cancer (AJCC) system was used for staging. ${ }^{4}$ Physical examination, mammography, and breast ultrasound were performed in all patients for locoregional staging. As recommended by NCCN, blood tests and conventional imaging were performed to screen for systemic disease. ${ }^{7}$ This staging method was defined as conventional CPS (c-CPS). Staging with FDG-PET/CT in addition to conventional methods was defined as PET-CPS.

All tumours were evaluated immunohistochemically for ER, PR, Her2, Ki-67 status, and histological grade. The scoring recommendation of the American Society of Clinical Oncology/College of American Pathologists was used to evaluate ER, PR and Her2 status. $^{12,13}$

Patients who were: examined before NAT with FDG-PET /CT were included in this study. Exclusion criteria were: being younger than 18 years, presence of metastatic disease before NAT, and not being evaluated with FDG-PET/CT before NAT. Five patients, who were not evaluated with PET-CT before NAT; and 12 patients with metastatic disease detected by conventional methods or PET-CT, were excluded from the study. All data were recorded from individual patient's health records.
The age of patients was investigated using visual (histograms, and probability plots) and Shapiro-Wilk's test to determine whether or not they were normally distributed. Clinical primary tumour (cT) and regional lymph node (cN) stages were recorded, according to both conventional methods and by adding FDG-PET/CT to conventional methods. Anatomical, cCPS, and pet-CPS stages of each patient were evaluated. The agreement between the anatomical stage and c-CPS was evaluated with the Kappa test. Wilcoxon test was used to evaluate whether there was a significant change in CT, CN, and CPS stages with the addition of FDG-PET/CT to conventional methods. If a significant difference was found, using the Wilcoxon test, the re-comparison was planned for each cT, cN, and c- CPS subgroup to determine from which subgroup the significance emanated. Significance for subgroups was obtained by the Wilcoxon test/number of subgroups (Bonferroni correction). All statistical tests were 2-sided with a significance level of $p<0.05$. The data were analysed using the Statistical Package for the Social Sciences, Statistics V.22 (IBM Corp, Armonk, NY).

\section{RESULTS}

A total of 121 patients with BC were eligible and included in this trial (age: $27-84$ years, median: 46$)$. Seventy-three (60.3\%) of the patients were premenopausal. The distributions of ER-positive $(E R+)$, PR-positive $(\mathrm{PR}+)$, and Her2-positive (Her2+) tumours were $81(66.9 \%), 73(60.3 \%), 48(39.7 \%)$ in all patients, respectively. The distribution of histologic grade was as follows: grade 1, 2, and 3 in 7 (5.8\%), 35 (28.9\%), 79 (65.3\%), respectively. The $\mathrm{CT}$ and $\mathrm{cN}$ distributions determined by conventional methods and by adding FDG-PET/CT to these methods are summarised in Table I. There was a significant change in the $\mathrm{cN}$ stages when evaluated by FDG-PET /CT for the $c N$ stage ( $p$ $<0.001$ ). There was no significant difference between the two methods for the $c T$ stage ( $p=0.180$ ). It was evaluated from which cN subgroups this change originated. The change was detected in 5/16 (6.3\%, $p=0.034), 14 / 71(19.7 \%, p<0.01)$, $15 / 30(50 \%, p<0.01), 0 / 4(0 \%, p=1)$ for patients with $\mathrm{cN} 0,1,2$, 3; respectively.

The distribution of anatomical stage by conventional methods was 2 (1.7\%), 20 (16.5\%), 46 (38\%), 28 (23.1\%), 21 (17.4\%), 4 (3.3\%); for IA, IIA, IIB, IIIA, IIIB, IIIC; respectively. The C-CPS and PET-CPS distributions are shown in Table II. A fair agreement was detected between anatomical and c-CPS stages distributions (kappa $=0.274, \mathrm{p}<0.001$ ).

The stages determined by anatomical staging were changed in 74 (61.1\%) ofall patients when evaluated by c-CPS. There was a downstaging in $46(62.2 \%)$ and an upstaging in 28 (37.8\%) patients. Substantial agreement was detected between the c-CPS and PETCPS (kappa: 0.74, p <0.001). An up-staging was detected with PET-CPS in 26/121 (21.5\%) patients who were initially evaluated by c-CPS. There was no downstaging with PET-CPS in any of the patients. The change between the $c$-CPS and PET-CPS was significant ( $p<0.001$ ). The distributions of the stages according to PETCPS and c-CPS were presented in Figure 1. 
Table I: T and N stages, according to conventional methods and FDG-PET/CT adding to conventional methods.

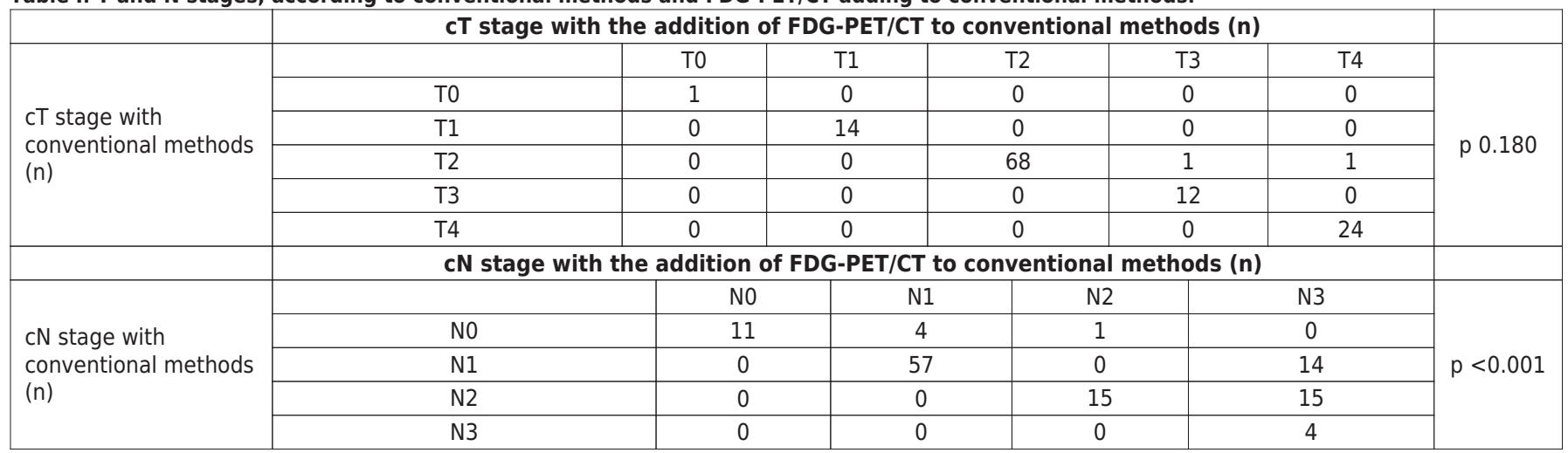

Table II: Distributions, of stages, according to C-CPSs and PET-CPSs.

\begin{tabular}{|c|c|c|c|c|c|c|c|c|c|}
\hline & \multicolumn{8}{|c|}{ pet-CPS(n) } & \multirow[b]{2}{*}{$\begin{array}{c}\text { Stage-changed patients, } \\
\text { n (\%) }\end{array}$} \\
\hline & & IB & IIA & IIB & IIIA & IIIB & IIIC & Total & \\
\hline \multirow[t]{8}{*}{$c-C P S(n)$} & $\mathrm{IA}$ & 0 & 1 & 0 & 0 & 0 & 0 & 1 & $0(0)$ \\
\hline & $\mathrm{IB}$ & 17 & 0 & 1 & 0 & 3 & 1 & 22 & $5 / 22(22.7)$ \\
\hline & IIA & 0 & 20 & 1 & 2 & 5 & 0 & 28 & $8 / 28(28.6)$ \\
\hline & IIB & 0 & 0 & 15 & 0 & 3 & 0 & 18 & $3 / 18(37.5)$ \\
\hline & IIIA & 0 & 0 & 0 & 7 & 5 & 2 & 14 & $7 / 14(50)$ \\
\hline & IIIB & 0 & 0 & 0 & 0 & 20 & 2 & 22 & $2 / 22(9.1)$ \\
\hline & IIIC & 0 & 0 & 0 & 0 & 0 & 16 & 16 & $0 / 16(0)$ \\
\hline & Total & 17 & 21 & 17 & 9 & 36 & 21 & 121 & $25 / 121(20.7)^{*}$ \\
\hline
\end{tabular}

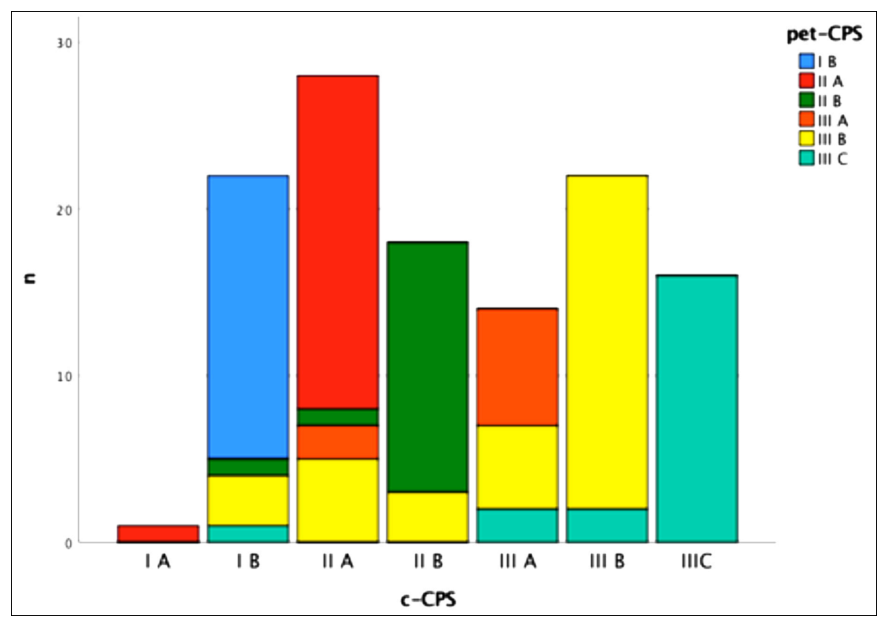

Figure 1: Distrubition of C-CPS and PET-CPS.

C-CPS: Clinical prognostic stage with conventional methods; PET-CPS: Clinical prognostic stage with FDG-PET/CT adding to conventional methods;FDG-PET/CT: Fluoro 18-fluorodeoxyglucose positron emission tomography/computerised tomography.

\section{DISCUSSION}

NAT is frequently preferred in the treatment of BC. Breast-conserving surgery and/or more limited intervention in the axilla becomes possible with NAT in many patients. Moreover, the pathological complete response (pCR) obtained with NAT is an important prognostic marker. ${ }^{14}$ Today, the most commonly used chemotherapy combination for NAT is anthracycline and taxane-based regimens. The PCR rates were increased by an escalation of treatment through adding dual anti-Her2 blockade in Her2+ patients and, platinum in TN BC patients. ${ }^{15,16}$ On the other hand, patients who had low-risk Her2 + BC ( $<3 \mathrm{~cm}$ tumours and node-negative) demonstrated striking results with a 7-year disease-free survival of $93 \%$ and, overall survival of $95 \%$ despite de-escalation of treatment to 12-week-paclitaxel and 1-year trastuzumab. ${ }^{17}$ Similarly, satisfactory survival results were obtained with the combination of docetaxel and cyclophosphamide in low-risk Her2-negative BC patients. ${ }^{17}$ These studies in the adjuvant setting raise the question of whether similar PCR rates and survival outcomes can be achieved with less chemotherapy and even without cytotoxic chemotherapy (e.g., dual anti-Her 2 blockade) in the neoadjuvant setting. ${ }^{6,18,19}$

In the light of available data, many different combinations of chemotherapy and targeted therapy can be used in NAT. ${ }^{7}$ Given that many NAT alternatives exist, and not every BC patient has the same risk of recurrence, choosing the most appropriate treatment for each patient is a critical decision. The stage of the disease is one of the most important factors besides the biological characteristics of the tumour in this decision.

The CPS defined by AJCC 8th edition provides more accurate prognostic information than anatomical staging. ${ }^{5}$ Prognostic prediction, based on the anatomic stage alone, may lead to under- or over-treatment. For this reason, we believe that a NAT plan, based on the recurrence risk for each patient, may 
be more rational. In this context, we think that it may be appropriate to evaluate the role of CPS in future studies on personalized NAT selection. Therefore, in this study, the authors aimed to investigate whether performing FDG-PET/CT in addition to conventional imaging studies would change the CPS stage in patients for whom NAT was planned.

In this study, the change in $\mathrm{cN}$ staging with the addition of FDG-PET/CT to conventional methods was significant ( $p$ $<0.001)$. This significance was due to upstaging in N1 and N2 disease ( $p<0.001)$. Fourteen $(19.7 \%)$ out of 71 patients, who were N1 at baseline; and 15 (50\%) out of 30 patients, who were N2 at baseline, were staged as N3 with FDGPET/CT. The authors detected that 5 out of 16 patients with $\mathrm{NO}$ at baseline were node-positive by FDG-PET/CT ( $p=$ 0.034). Although not statistically significant due to Bonferroni correction, this may be due to the small number of patients with $\mathrm{NO}$ disease. The low number of patients in this group may be due to the retrospective design of this study. However, considering that nodal disease was detected with FDG-PET/CT in 5/16 (31.3\%) patients with cN0 disease with conventional methods, this may be clinically significant. It is necessary to investigate how FDG-PET/CT changes nodal staging in more patients with cNO disease.

There was a significant change in the CPS in 25/121 (20.7\%) patients when FDG-PET/CT was performed in addition to conventional imaging $(p<0.001)$. When subgroups were examined, there was no statistical significance due to the small number of patients per stage. However, 22.7\% upstaging was found even in C-CPS stage-IB patients. This finding is clinically important. For this reason, it may be considered to add FDG-PET/CT to conventional imaging studies for staging in BC patients receiving NAT. The fact,in which stages PET-CT would be useful, should be evaluated in studies conducted with more patients.

The authors could not find any other study in the literature evaluating the effect of adding FDG-PET/CT to conventional methods on CPS. The change in N stages was due to higher rates of N3 disease with FDG-PET/CT in N1 and N2 patients with conventional methods. There was no significant change in the CT stages of the patients. This finding, which is consistent with previous studies, is a consequence of better detection of the internal mammary node, supraclavicular node, and infraclavicular node with FDG-PET /CT. ${ }^{9,20}$ In a previous study, pathological confirmation was done by a biopsy performed for supraclavicular lymph node metastases detected by conventional methods or FDG-PET/CT in BC patients, and sensitivity was $91.7 \%$, while accuracy was $99.8 \%$ with FDG-PET/CT alone. ${ }^{10}$ Pathologic confirmation of internal mammary lymph nodes detected with FDG-PET /CT in BC patients also revealed a very high probability of malignant involvement. ${ }^{21}$ Considering these data in the literature, although biopsy was not done for N3 lymph nodes detected on FDG-PET/CT, this is a negligible limitation because of the low probability of false-positive FDG uptake in supraclavicular, infraclavicular, and internal mammary lymph nodes.

Due to the retrospective nature of this study, there were no strict criteria in the selection of patients to be given NAT. Hence, there may be more biologically aggressive tumours in this study that are expected to benefit more from NAT. This may have affected the results of this study. BC patients with more aggressive tumour biology may be more likely to have internal mammary lymph node involvement. ${ }^{22}$ For this reason, it will be more valuable to evaluate the role of FDG-PET/CT in determining the stage of CPS with patient populations separated by molecular subtypes in future studies.

It is clear that individualised treatment will be increasingly preferred according to the biological characteristics of the tumour, its stage, and the patient's preferences in both neoadjuvant and adjuvant settings in the treatment of BC. The most important factor in deciding to de-escalate or escalate NAT will be the risk of recurrence. The main factors determining the risk of recurrence are molecular subtype, axillary lymph node involvement, tumour size, and grade. These factors are covered by CPS.

\section{CONCLUSION}

According to the present results, performing FDG-PET/CT in the evaluation of CPS significantly increases the stage in BC patients with planned NAT. The prognostic significance of this finding should be investigated in more comprehensive studies.

\section{ETHICAL APPROVAL:}

This study was approved by the Haydarpasa Numune Training and Research Hospital Institutional Review Board (Approval No. 2020/248).

\section{PATIENT'S CONSENT:}

Because this study was retrospective, the patients' consents were waived.

\section{CONFLICT OF INTEREST:}

The authors declared no conflict of interest.

\section{AUTHORS' CONTRIBUTION:}

ETS: Supervision, data acquisition for the work, the acquisition, analysis, interpretation of data for the work, original draft preparation.

EC: Data acquisition, revising it critically for important intellectual content, original draft preparation.

EA: Data acquisition, revising it critically for important intellectual content.

SG: Data collection and processing, critical review.

FA, MS: Data acquisition.

\section{REFERENCES}

1. Sung H, Ferlay J, Siegel RL, Laversanne M, Soerjomataram I, Jemal A, et al. Global Cancer Statistics 2020: GLOBOCAN Estimates of Incidence and Mortality Worldwide for 36 Cancers in 185 Countries. CA: A Cancer J Clin 2021; 
71(3):209-49. doi: 10.3322/caac. 21660.

2. Chan CWH, Law BMH, So WKW, Chow KM, Waye MMY. Novel strategies on personalised medicine for breast cancer treatment: An Update. Int J Mol Sci 2017; 18(11) doi: 10.3390/ijms18112423.

3. Edge SB, Compton CC. The American Joint committee on cancer: the 7th edition of the AJCC cancer staging manual and the future of TNM. Ann Surg Oncol 2010; 17(6):1471-4. doi: 10.1245/s10434-010-0985-4.

4. Gabriel N. Hortobagyi James L, Carl JD'Orsi C, Edge S, Elizabeth A, Mittendorf, et al. Breast Cancer. In: Amin MB, editor. AJCC Cancer Staging Manual 2017.

5. Wang $M$, Chen $H$, Wu K, Ding A, Zhang M, Zhang P. Evaluation of the prognostic stage in the 8th edition of the American joint committee on cancer in locally advanced breast cancer: An analysis based on SEER 18 database. Breast 2018; 37:56-63. doi: 10.1016/j.breast.2017.10.011.

6. Werutsky G, Rosa ML. De-escalation of neoadjuvant therapy for HER2-positive early breast cancer: an overview. Ann Palliat Med 2020; 9(4):1352-7. doi: 10.21037/apm-20-1035.

7. NCCN Clinical Practice Guidelines in Oncology. Breast Cancer. Version 4.2021 [Available from: http://www.nccn.org/professionals/physician_gls/pdf/breast. pdf.

8. Cardoso F, Kyriakides S, Ohno S, Penault-Llorca F, Poortmans P, Rubio IT, et al. Early breast cancer: ESMO clinical practice guidelines for diagnosis, treatment and followupdagger. Ann Oncol 2019; 30(8):1194-220.

9. Yararbas U, Avci NC, Yeniay L, Argon AM. The value of $18 \mathrm{~F}$ FDG PET/CT imaging in breast cancer staging. Bosn J Basic Med Sci 2018; 18(1):72-9. doi: 10.17305/bjbms.2017.2179.

10. Lee J, Park HY, Kim WW, Park CS, Lee RK, Kim HJ, et al. Value of accurate diagnosis for metastatic supraclavicular lymph nodes in breast cancer: Assessment with neck US, CT, and 18F-FDG PET/CT. Diagn Interv Radiol 2021; 27(3):323-8. doi: 10.5152/dir.2021.20190.

11. Koolen BB, Vrancken Peeters MJ, Aukema TS, Vogel WV, Oldenburg HS, van der Hage JA, et al. 18F-FDG PET/CT as a staging procedure in primary stage II and III breast cancer: Comparison with conventional imaging techniques. Breast Cancer Res Treat 2012; 131(1):117-26.

12. Hammond MEH, Hayes DF, Dowsett M, Allred DC, Hagerty $\mathrm{KL}$, Badve S, et al. American society of clinical oncology/college of american pathologists guideline recommendations for immunohistochemical testing of estrogen and progesterone receptors in breast cancer. J Clin Oncol 2010; 28(16):2784-95. doi: 10.1200/JCO.2009.25.6529.

13. Wolff AC, Hammond ME, Hicks DG, Dowsett M, McShane LM, Allison $\mathrm{KH}$, et al. Recommendations for human epidermal growth factor receptor 2 testing in breast cancer: American society of clinical oncology/college of American pathologists clinical practice guideline update. J Clin Oncol 2013; 31(31):3997-4013. doi: 10.1200/JCO.2013.50.9984.

14. Spring LM, Fell G, Arfe A, Sharma C, Greenup R, Reynolds KL, et al. Pathologic Complete response after neoadjuvant chemotherapy and impact on breast cancer recurrence and survival: A comprehensive meta-analysis. Clin Cancer Res 2020; 26(12):2838-48. doi: 10.1158/1078-0432.CCR-19-3492.

15. Wang C, Chen J, Xu X, Hu X, Kong D, Liang G, et al. Dual HER2 blockade in neoadjuvant treatment of HER2 + breast cancer: A meta-analysis and review. Technoin Cancer Res Treatment 2020; 19:153303382096072. doi: 10.1177/1533 033820960721.

16. Li ZY, Zhang Z, Cao XZ, Feng Y, Ren SS. Platinum-based neoadjuvant chemotherapy for triple-negative breast cancer: A systematic review and meta-analysis. J Int Med Res 2020; 48(10):030006052096434. doi: 10.1177/03000 60520964340.

17. Nitz U, Gluz O, Christgen M, Kates RE, Clemens M, Malter W, et al. Reducing chemotherapy use in clinically high-risk, genomically low-risk pNO and pN1 early breast cancer patients: Five-year data from the prospective, randomised phase 3 West German Study Group (WSG) PlanB trial. Breast Cancer Res Treat 2017; 165(3):573-83. doi: 10.1007/s10549-017-4358-6.

18. Guarneri V, Dieci MV, Bisagni G, Frassoldati A, Bianchi GV, De Salvo GL, et al. De-escalated therapy for HR+/HER2+ breast cancer patients with Ki67 response after 2-week letrozole: Results of the PerELISA neoadjuvant study. Ann Oncol 2019; 30(6):921-6. doi: 10.1093/annonc/mdz055.

19. Li WP, Zhu T, Hu MX, Yang M, Ji F, Gao HF, et al. Comparison of the efficacy and safety of the EC-T (epirubicin/cyclophosphamide followed by docetaxel) and TCb (docetaxel/carboplatin) neoadjuvant regimens in early TOP2A-normal stage II-III breast cancer. Neoplasma 2020; 67(6):1409-15. doi: 10.4149/neo_2020_200130N96.

20. Groheux D, Hindie E. Breast cancer: Initial workup and staging with FDG PET/CT. Clin Transl Imaging 2021; 1-11. doi: 10.1007/s40336-021-00426-z.

21. Wang CL, Eissa MJ, Rogers JV, Aravkin AY, Porter BA, Beatty JD. 18F-FDG PET/CT-Positive internal mammary lymph nodes: Pathologic correlation by ultrasound-guided fineneedle aspiration and assessment of associated risk factors. Am J Roentgenol 2013; 200(5):1138-44. doi: 10.2214/AJR.12.8754.

22. Singh S, Ramani SK, Rastogi A, Thakur MH. Incidence of internal mammary node in locally advanced breast cancer and its correlation with metastatic disease: A retrospective observational study. Br J Radiol 2019; 92(1103):20190098. doi: 10.1259/bjr.20190098. 